\title{
Tolerogenic Dendritic Cells as a Promising Antigen-Specific Therapy in the Treatment of Multiple Sclerosis and Neuromyelitis Optica From Preclinical to Clinical Trials
}

\author{
Georgina Flórez-Grau',2, Irati Zubizarreta ${ }^{2}$, Raquel Cabezón ${ }^{1,2}$, Pablo Villoslada ${ }^{2}$ \\ and Daniel Benitez-Ribas ${ }^{1 *}$ \\ 'Department of Immunology, Hospital Clinic i Provincial, Barcelona, Spain, ${ }^{2}$ Neuroimmunology Group, Institut \\ d'Investigacions Biomèdiques August Pi i Sunyer (IDIBAPS), Barcelona, Spain
}

OPEN ACCESS

Edited by: Natalio Garbi, Universität Bonn, Germany

Reviewed by:

Muriel Moser,

Free University of Brussels,

Belgium

Isis Ludwig-Portugall, Universität Bonn, Germany

*Correspondence: Daniel Benitez-Ribas dbenitezr@clinic.cat

Specialty section: This article was submitted to Immunological Tolerance and Regulation, a section of the journal

Frontiers in Immunology

Received: 30 October 2017 Accepted: 09 May 2018

Published: 31 May 2018

Citation:

Flórez-Grau G, Zubizarreta I,

Cabezón R, Villoslada P and Benitez-Ribas D (2018) Tolerogenic

Dendritic Cells as a Promising

Antigen-Specific Therapy in the

Treatment of Multiple Sclerosis and Neuromyelitis Optica From Preclinical to Clinical Trials.

Front. Immunol. 9:1169. doi: 10.3389/fimmu.2018.01169
The identification of activated T-lymphocytes restricted to myelin-derived immunogenic peptides in multiple sclerosis (MS) and aquaporin-4 water channel in neuromyelitis optica (NMO) in the blood of patients opened the possibility for developing highly selective and disease-specific therapeutic approaches. Antigen presenting cells and in particular dendritic cells (DCs) represent a strategy to inhibit pro-inflammatory $T$ helper cells. DCs are located in peripheral and lymphoid tissues and are essential for homeostasis of $T$ cell-dependent immune responses. The expression of a particular set of receptors involved in pathogen recognition confers to DCs the property to initiate immune responses. However, in the absence of danger signals different DC subsets have been revealed to induce active tolerance by inducing regulatory $T$ cells, inhibiting pro-inflammatory $\mathrm{T}$ helper cells responses or both. Interestingly, several protocols to generate clinical-grade tolerogenic DC (Tol-DC) in vitro have been described, offering the possibility to restore the homeostasis to central nervous system-related antigens. In this review, we discuss about different DC subsets and their role in tolerance induction, the different protocols to generate Tol-DCs and preclinical studies in animal models as well as describe recent characterization of Tol-DCs for clinical application in autoimmune diseases and in particular in MS and NMO patients. In addition, we discuss the clinical trials ongoing based on Tol-DCs to treat different autoimmune diseases.

Keywords: tolerogenic dendritic cells, dendritic cells, immunotherapy, immunosuppression, multiple sclerosis, Neuromyleitis optica

\section{INTRODUCTION}

Multiple Sclerosis (MS) is a chronic autoimmune inflammatory disease affecting the central nervous system (CNS) (1). Nowadays, there are 2.3 million affected people worldwide, being the most frequent age of diagnosis between 20 and 40 years old (2). Additionally, the studies determine that MS is more frequent in women and in northern locations. There are different subtypes of MS which

Abbreviations: APCs, antigen presenting cells; APL, altered peptide ligand; AQP4, aquaporin-4; CNS, central nervous system; DCs, dendritic cells; Mo-DCs, monocyte-derived DCs; MS, multiple sclerosis; NMO, neuromyelitis optica; PPMS, primaryprogressive MS; RA, rheumatoid arthritis; RRMS, relapsing-remitting MS; SPMS, secondary-progressive MS; Tol-DCs, tolerogenic DCs. 
are based on their clinical phenotype (3). These subtypes are: The primary-progressive MS (PPMS) which is a disabling subtype from the beginning, the relapsing-remitting type (RRMS) that is characterized by clinical relapses without progression of disability and finally, the secondary-progressive subtype that appears about 20 years after RRMS.

The MS diagnosis is summarized in the revised $2010 \mathrm{Mc}$ Donald criteria which is included in Table 1 (4). Although the cause of the immune deregulation is unknown, there are evidences that implicate Th1 and Th17 lymphocytes in the pathophysiology of MS (5-10). Furthermore, it was supported by studies performed in experimental models of MS either knocking out or blocking using monoclonal antibodies for IL-17 or IL-23 resulted in a suppression of the activity of this disease $(11,12)$. Other authors have described that memory T-cells are activated in the periphery by different processes that can be promoted by environmental or genetic factors. These activated cells cross the blood-brain barrier, penetrate to CNS where they are locally reactivated $(9,13)$.

First-line therapies for MS include injectable treatments such as IFN- $\beta$, and glatiramer as well as oral therapies such as teriflunomide and dimethyl-fumarate. Second-line therapies include fingolimod, and the intravenous natalizumab, which present higher levels of efficacy in reducing the relapse rate; however, it has potential severe side effects. Moreover, Alentuzumab, Cladribine, and Ocrelizumab were recently added as approved therapies, and they are in progress of being defined in the pyramid of the MS therapy. All these mentioned treatments are systemic immunomodulatory or immunosuppressive treatments with risks of adverse events.

Neuromyelitis optica (NMO) is an inflammatory disease affecting the CNS (14) with similar physiopathology as MS, but is considered an autoimmune astrocytopathy. NMO is a rare disease which presents with incidence between 0.05 and $0.4 / 100,000$ $(15,16)$. About $70 \%$ of the patients diagnosed with NMO shows the presence of anti-aquaporin-4 (AQP4) antibody as well as specific T-lymphocytes in the bloodstream or CSF which suggest the pro-inflammatory role of these cells (17). Importantly, the detection of anti-AQP4 antibodies is related with more severe disease (14). Recently, among seronegative patients, anti-(MOG) antibodies have been described as the pathological antibody (18). This disease has its own international consensus diagnostic criteria (19), defining the NMO spectrum disorder (NMOSD) concept (Table 2). Different MS drugs such as natalizumab or finolimob have been evaluated in NMO resulting in exacerbation of relapses (20). Immunomodulatory or immunossuppressant therapies are used for label in NMOSD (e.g., azathioprine, mycophenolate, cyclophosphamide, or rituximab) (21). Furthermore, several monoclonal antibodies are in clinical trials to evaluate their efficacy and safety, as tocilizumab, satralizumab, eculizumab, or aquapuromab for example (22). Based in the unmet need of achieving higher levels of efficacy and/or better safety profile, antigen-specific therapies are being considered as a potential treatment for MS and NMO (19).

\section{DENDRITIC CELLS (DCs)}

Dendritic cells act as a link between innate and adaptive immune responses. Their main function is to capture and process exogenous antigens in the peripheral tissues to present them to T-cells after migration to the draining lymph nodes. In addition, they polarize immune responses by promoting both pro- and antiinflammatory immune responses depending on the presence of danger signals associated to the antigens (Figure 1) $(24,25)$.

TABLE 1 | 2010 Mc Donald criteria for multiple sclerosis (MS) diagnosis (4).

\begin{tabular}{|c|c|}
\hline Clinical presentation & Additional data needed for MS diagnosis \\
\hline $\begin{array}{l}2 \text { or more attacks; objective clinical evidence } \\
\text { of } 2 \text { or more lesions or objective clinical } \\
\text { evidence of } 1 \text { lesion with reasonable historical } \\
\text { evidence of a prior attack }\end{array}$ & None \\
\hline $\begin{array}{l}2 \text { or more attacks; objective clinical } \\
\text { evidence of } 1 \text { lesion }\end{array}$ & $\begin{array}{l}\text { Dissemination in space, demonstrated by: } \\
1 \text { or more T2 lesions in at least } 2 \text { of } 4 \text { MS-typical regions of the central nervous system (CNS) (periventricular, } \\
\text { juxtacortical, infratentorial, or spinal cord); or await a further clinical attack implicating a different CNS site }\end{array}$ \\
\hline $\begin{array}{l}1 \text { attack; objective clinical evidence } \\
\text { of } 2 \text { or more lesions }\end{array}$ & $\begin{array}{l}\text { Dissemination in time, demonstrated by: } \\
\text { simultaneous presence of asymptomatic gadolinium-enhancing and non-enhancing lesions at any time; } \\
\text { or A new T2 and/or gadolinium-enhancing lesion(s) on follow-up MRI, irrespective of its timing with reference } \\
\text { to a baseline scan; or await a second clinical attack }\end{array}$ \\
\hline $\begin{array}{l}1 \text { attack; objective clinical evidence } \\
\text { of } 1 \text { lesion (clinically isolated syndrome) }\end{array}$ & $\begin{array}{l}\text { Dissemination in space and time, demonstrated by: } \\
\text { for DIS: } 1 \text { or more T2 lesion in at least } 2 \text { of } 4 \text { MS-typical regions of the CNS (periventricular, juxtacortical, } \\
\text { infratentorial, or spinal cord); or await a second clinical attack implicating a different CNS site; and } \\
\text { for DIT: simultaneous presence of asymptomatic gadolinium-enhancing and non-enhancing lesions } \\
\text { at any time; or a new T2 and/or gadolinium-enhancing lesion(s) on follow-up MRI, irrespective of its } \\
\text { timing with reference to a baseline scan; or Await a second clinical attack }\end{array}$ \\
\hline $\begin{array}{l}\text { Insidious neurological progression } \\
\text { suggestive of MS (PPMS) }\end{array}$ & $\begin{array}{l}\text { 1 year of disease progression (retrospectively or prospectively determined) plus } 2 \text { of } 3 \text { of the following criteria: } \\
\text { 1. evidence for DIS in the brain based on } 1 \text { or more T2 lesions in the MS-characteristic (periventricular, } \\
\text { juxtacortical, or infratentorial) regions } \\
\text { 2. evidence for DIS in the spinal cord based on } 2 \text { or more T2 lesions in the cord } \\
\text { 3. positive CSF (isoelectric focusing evidence of oligoclonal bands and/or elevated IgG index) }\end{array}$ \\
\hline
\end{tabular}


TABLE 2 | Neuromyelitis optica spectrum disorder diagnostic criteria from Ref. (23).

Diagnostic criteria for NMO spectrum disorder (NMOSD) with aquaporin-4 (AQP4)-lgG

1. At least 1 core clinical characteristic.

2. Positive test for AQP4-IgG using best available detection method (cell-based assay strongly recommended).

3. Exclusion of alternative diagnoses.

Diagnostic criteria for NMOSD without AQP4-IgG or NMOSD with unknown AQP4-lgG status

1. At least 2 core clinical characteristics occurring as a result of one or more clinical attacks and meeting all of the following requirements:

(a) At least 1 core clinical characteristic must be optic neuritis, acute myelitis with LETM, or area postrema syndrome

(b) Dissemination in space (2 or more different core clinical characteristics)

(c) Fulfillment of additional MRI requirements, as applicable

2. Negative tests for AQP4-lgG using best available detection method, or testing unavailable

3. Exclusion of alternative diagnoses

Core clinical characteristics.

1. Optic neuritis.

2. Acute myelitis.

3. Area postrema syndrome: unexplained hiccups or nausea and vomiting

4. Acute brainstem syndrome.

5. Symptomatic narcolepsy or acute diencephalic clinical syndrome with NMOSD-typical diencephalic MRI lesions.

6. Symptomatic cerebral syndrome with NMOSD-typical brain lesions.

Additional MRI requirements for NMOSD without AQP4-IgG and NMOSD with unknown AQP4-lgG status.

1. Acute optic neuritis: requires brain MRI showing (a) normal findings or only nonspecific white matter lesions, OR (b) optic nerve MRI with T2-hyperintense lesion or T1-weighted gadolinium-enhancing lesion extending over 1/2 optic nerve length or involving optic chiasm.

2. Acute myelitis: intramedullary MRI lesion extending over 3 contiguous segments (LETM) OR 3 contiguous segments of focal spinal cord atrophy in patients with history compatible with acute myelitis.

3. Area postrema syndrome: dorsal medulla/area postrema lesions.

4. Acute brainstem syndrome: periependymal brainstem lesions.

Dendritic cells are located in peripheral tissues (skin and mucosa) and remain in an immature state (iDCs) until they interact with the antigens. After cells activation, DCs initiate a maturation process in which mature DCs (mDCs) lose capacities for antigen uptake in favor of acquiring stimulatory properties for the activation of naïve T-cells and the development of effector T-cells (27). Maturation process involves different processes and physiological changes in DCs, which are illustrated in Figure 2 (28).

Due to their immunological functions and the availability of clinical-grade reagents, immunogenic DCs have been safely used in clinical trials to potentiate immune response against tumors or infectious diseases (30). However, only a few studies recently published have taken advantage of their specific tolerogenic properties to treat Type 1 diabetes, rheumatoid arthritis (RA) and Crohn's disease patients $(25,31,32)$.

\section{HUMAN DCs SUBSETS}

Dendritic cells can be sub-classified based on anatomical location, origin, and function. In humans, different DC subsets have been identified in blood, spleen and skin and in non-lymphoid tissues.

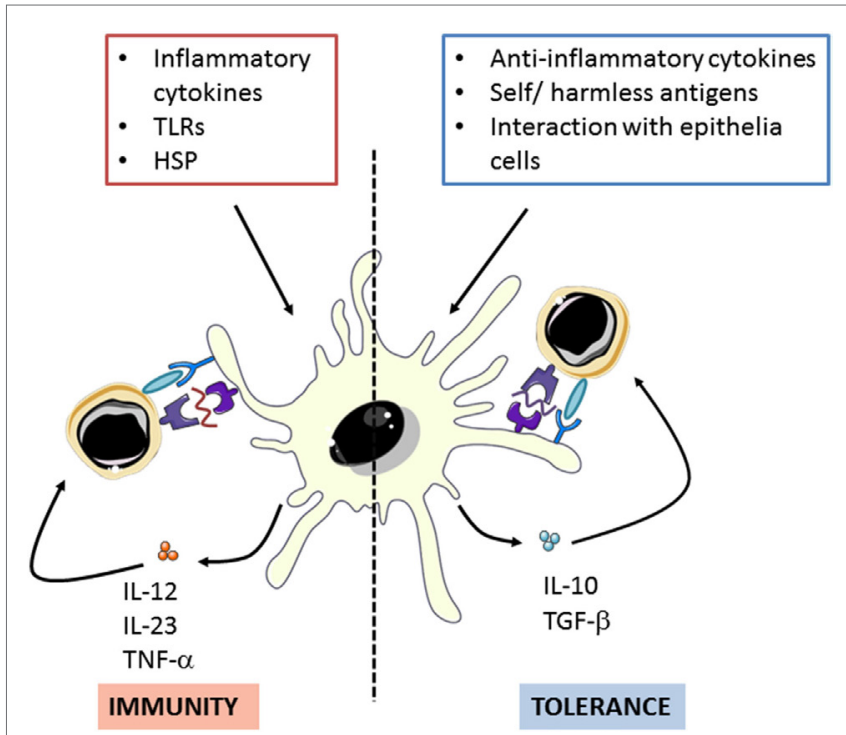

FIGURE 1 | Dendritic cells can polarize immune response though promote both pro- and anti-inflammatory activities in response to different stimuli. Adapted from: O'Neil et al. (26). TLRs: toll-like receptors, HSP: Heat shock proteins.

Each DC subset presents different specialization in T-cell priming and induction of immune responses, although their functions can partially overlap (33).

In peripheral blood, DCs that express Human Leukocyte Antigen-antigen D Related (HLA-DR) and lineage negative fraction are divided into two main groups: conventional myeloid DCs (cDCs) and non-conventional plasmacytoid DCs (pDC). Within myeloid DCs two main subsets have been identified based on their surface marker expression: CD1c/BDCA-1 cDCs and CD141/BDCA-3 cDCs. However, recently new DC subset classification has been described (CD16 and DC5) (23). Circulating DCs represent a little fraction of total circulating peripheral blood mononuclear cells (PBMCs) as they account for less than $1 \%$ of PBMCs $(24,34)$.

In the skin two different subsets of DCs can be found. Langerhans cells (LCs) which contributes to immune surveillance and CD14 DCs, which are involved in tolerance induction $(35,36)$.

From all the different DC subsets above mentioned, the BDCA-1, pDCs, LCs, and CD14 have been described to generate both immunogenic and suppressive functions (Figure 3). BDCA-1 have the capacity to produce IL-10 in response to E. coli and potentially contribute to suppress immune responses. Recently, a particular subset of BDCA-1 (BDCA1-CD14 ${ }^{+}$) has been shown to act as immunosuppressive cells in certain types of tumor environment and may hamper anti-cancer DCs vaccines $(37,38)$ Moreover, in an steady state, pDCs are able to induce tolerogenic immune responses by inducing T-cell anergy and promoting T-reg cells development. They have been found to be infiltrated in tumors activating $\operatorname{Tr} 1$ cells $(33,39,40)$. LCs, apart from respond to intracellular pathogens and viruses under inflammatory conditions are in charge to maintain epidermal health and tolerance to commensals from the skin, while retaining the 


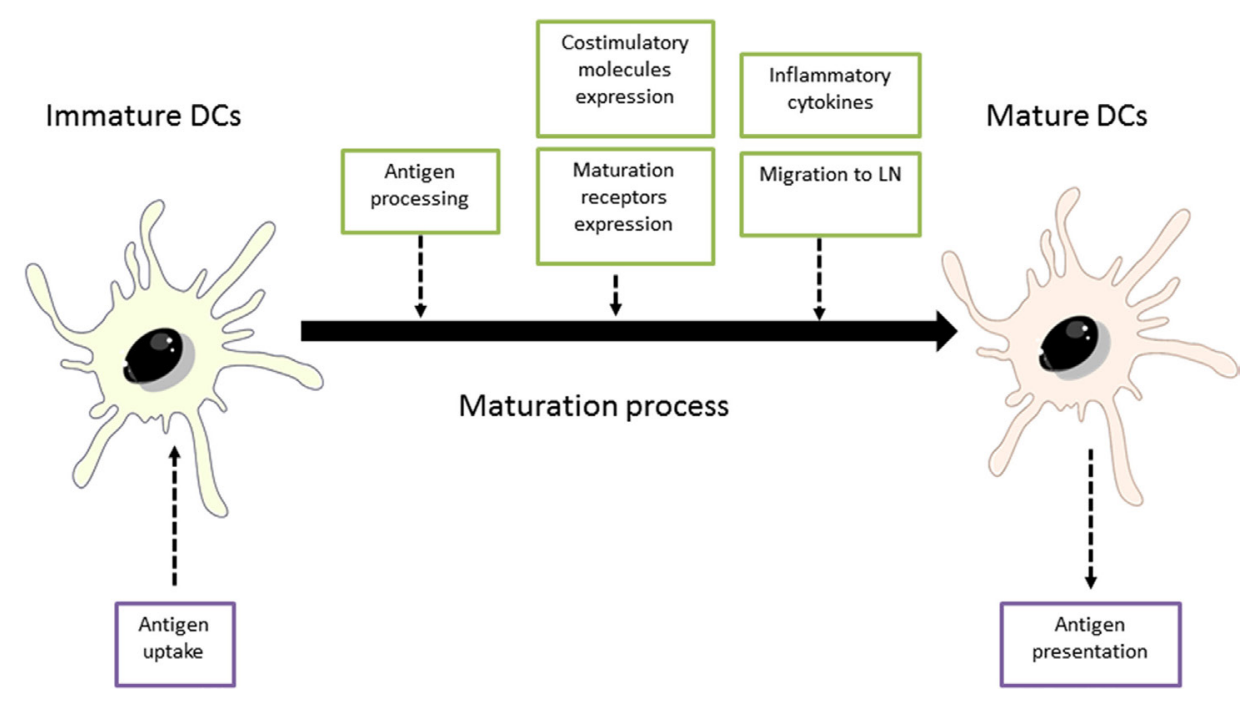

FIGURE 2 | Maturation process of dendritic cells (DCs). Adapted from: Steinman et al. (29). LN, lymph nodes.

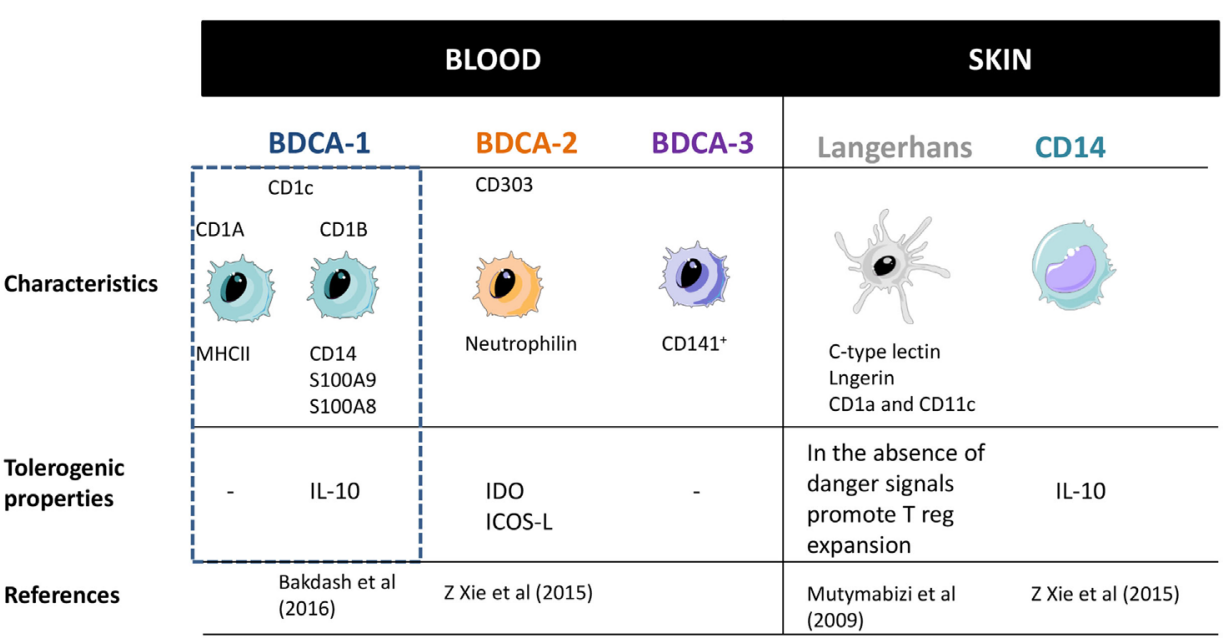

FIGURE 3 | Dendritic cells subsets classification and their main properties. Adapted from: Cohn et al. (37). PRRs, pattern recognition receptors.

ability to respond to selected pathogens (40-42). Finally CD14 DCs also have the ability to generate T-regs through the elevated IL-10 production $(43,44)$.

To sum up, BDCA-1, pDCs, LCs, and CD14 have been shown to present immunoregulatory effects. However, deeper characterization of this tolerogenic profile and mechanisms needs to be performed.

\section{TOLEROGENIC DCs (Tol-DCs) AND MECHANISMS OF TOLERANCE INDUCTION}

As described in the previous section, DCs play a crucial role in the initiation of immune responses and also in maintaining the immune tolerance. DCs present both foreign antigens as well as endogenous antigens derived from tissues. For this reason, the immune system is able to distinguish between innocuous and harmful antigens to avoid autoimmune or undesired immune responses (45). Several studies point that a key factor for DCs to initiate immunity or tolerance is the maturation stage of DCs (25). It is generally accepted that in absence of danger signals provided by infection or inflammation, DCs remain in an immature state which will induce tolerance by deleting or inducing apoptosis of self-antigen-specific T-cells $(25,46)$. However, other several mechanisms to explain how DCs induce tolerance have been proposed. Some authors have reported that low expression of MHC molecules and co-stimulatory receptors on DC surface fail to stimulate T-cells sufficiently, thus resulting in T-cell anergy (47-49). Currently, it has been demonstrated that the expression 
of single immunoglobulin IL-1 related receptor, which is lower in iDCs, has a role in maintain low levels of costimulatory molecules and in the regulation of T-reg cell expansion (50). Furthermore, it is well established that the expression of certain molecules such as $\mathrm{PD}-\mathrm{L} 1$ rather than promote activation signals to T-cells, they induce T-cell anergy $(28,51,52)$. Moreover, some authors demonstrated that suboptimal antigen presentation, together with indoleamine 2,3-dioxygenase (IDO) or Fas-L (CD95L) expression by DCs leads to inhibition of T-cell proliferation and T-cell deletion, respectively. Finally but not the least, the production of the potent anti-inflammatory cytokine IL-10 by DCs is crucial for peripheral tolerance induction. IL-10 acts on a wide variety of immune cells and it has been clearly involved in T-reg as well as $\operatorname{Tr} 1$ induction (38). In the steady state, peripheral T-reg cells rise from peripheral $\mathrm{CD}^{+}{ }^{+} \mathrm{CD} 25^{-} \mathrm{FOXP} 3^{-} \mathrm{T}$ cells that are exposed to antigen in the presence of transforming growth factor- $\beta$ as well as IL-10 without IL- 6 or IL- $1 \beta$, which promotes the up-regulation of FOXP3 (17) (Figure 4). Recent developments carried on by Agrawal et al., have shown that C-lectin receptor (CLEC-2) upregulation in DCs, is associated with T-reg induction. Moreover, they have also described that platelet growth factor is able to induce IL-10 production by DCs and in consequence T-reg cell induction (53).

In consequence, major efforts have been focused on in vitro generation of Tol-DCs. In this regard, different immunosuppressive drugs, such as corticosteroids, cyclosporine, tacrolimus, rapamycin, deoxyspergualin, vitamin D3 (vitD3), mycophenolate mofetil, and sanglifehrin A, have been successfully used to modulate DCs differentiation and function. Thus, several protocols that include the generation of monocyte-derived DCs in the presence of corticosteroids and a defined maturation cytokine cocktail (including TNF- $\alpha$, IL-1 $\beta$, IL-6, and PGE2) or lipopolysaccharide (LPS) activation in order to boost their tolerogenic properties, have been described to generate Tol-DCs in vitro $(54,55)$.

Tolerogenic DCs present an intermediate phenotype between iDCs and mDCs regarding costimulatory molecules, a pronounced shift toward anti-inflammatory versus pro-inflammatory cytokine production (high amounts of IL-10 versus low levels of IL-12p70 and IL-23) and a reduced capacity to stimulate T-cells response. In addition Tol-DCs present an increment of IL-10 production upon Gram-negative bacterial interaction which represents a relevant factor to induce tolerance due to the potent antiinflammatory role of IL-10 (Figure 5) (56-58).

The role of in vitro generated Tol-DCs as potential immunomodulatory and immunosuppressive agents have been evaluated by different groups $(44,60,61)$. The first experimental data to objectify the potential of human Tol-DC to induce tolerance in MS, was the induction of T-cell hyporesponsiveness by Tol-DC from MS patients. The results obtained shown that only Tol-DCs (vitD3) derived from RRMS patients, induced hyporesponsiveness in autologous antigen-specific T-cells restricted to myelinderived peptides and produced higher levels of IL-10 and reduced levels of TNF- $\alpha$ compared to healthy controls, making the tolerogenic potential of these autologous Tol-DCs may be an effective tool to re-establish tolerance in RRMS patients and set up the basis for the ongoing clinical trials (62). In addition, a critical consideration for Tol-DC application in immunotherapy is the phenotype stability once the cells are injected into the patients. It has been demonstrated that in vitro generated Tol-DCs have a stable tolerogenic profile after LPS stimulation as they produce higher amounts of IL-10 and as well as they are able to induce antigen-specific T-cell hyporesponsiveness $(58,63)$.

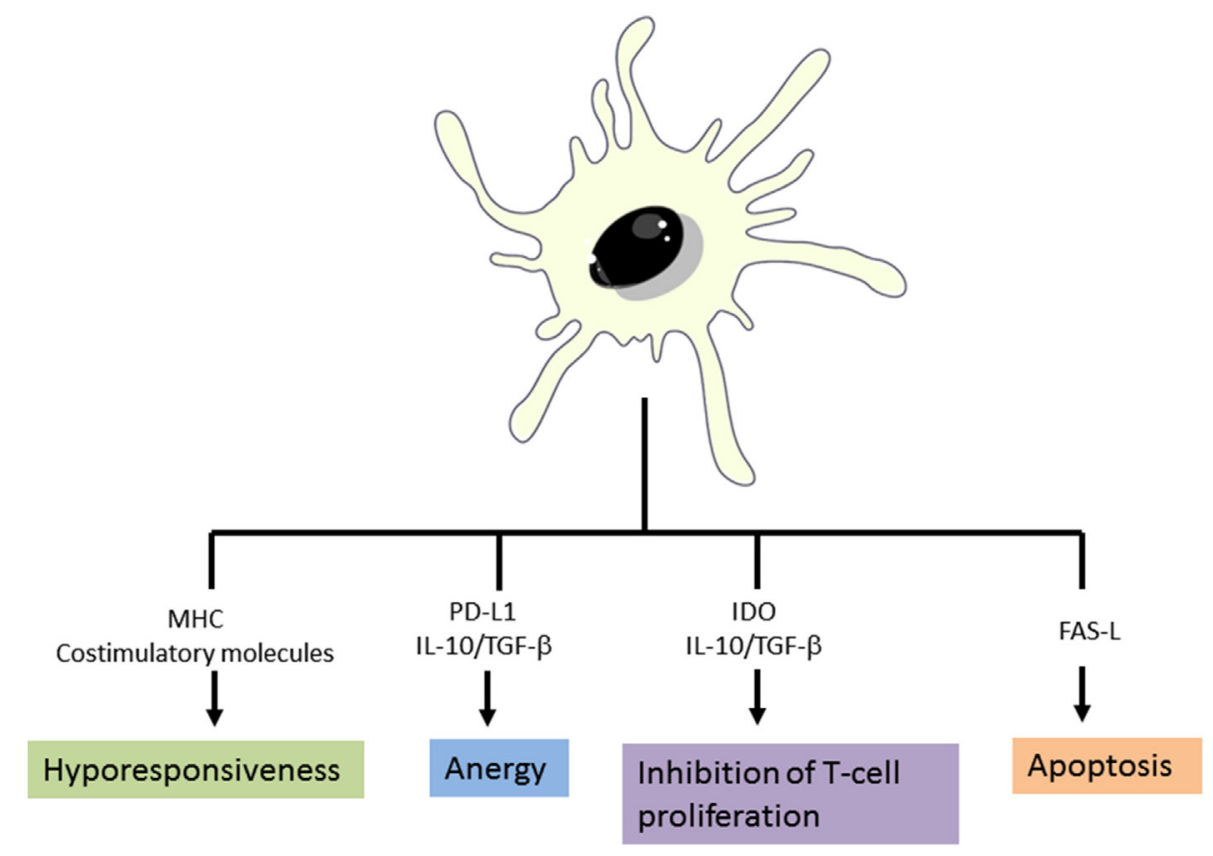

FIGURE 4 | Mechanisms of tolerance induction by dendritic cells. Adapted from: Cabezón et al. (30). 


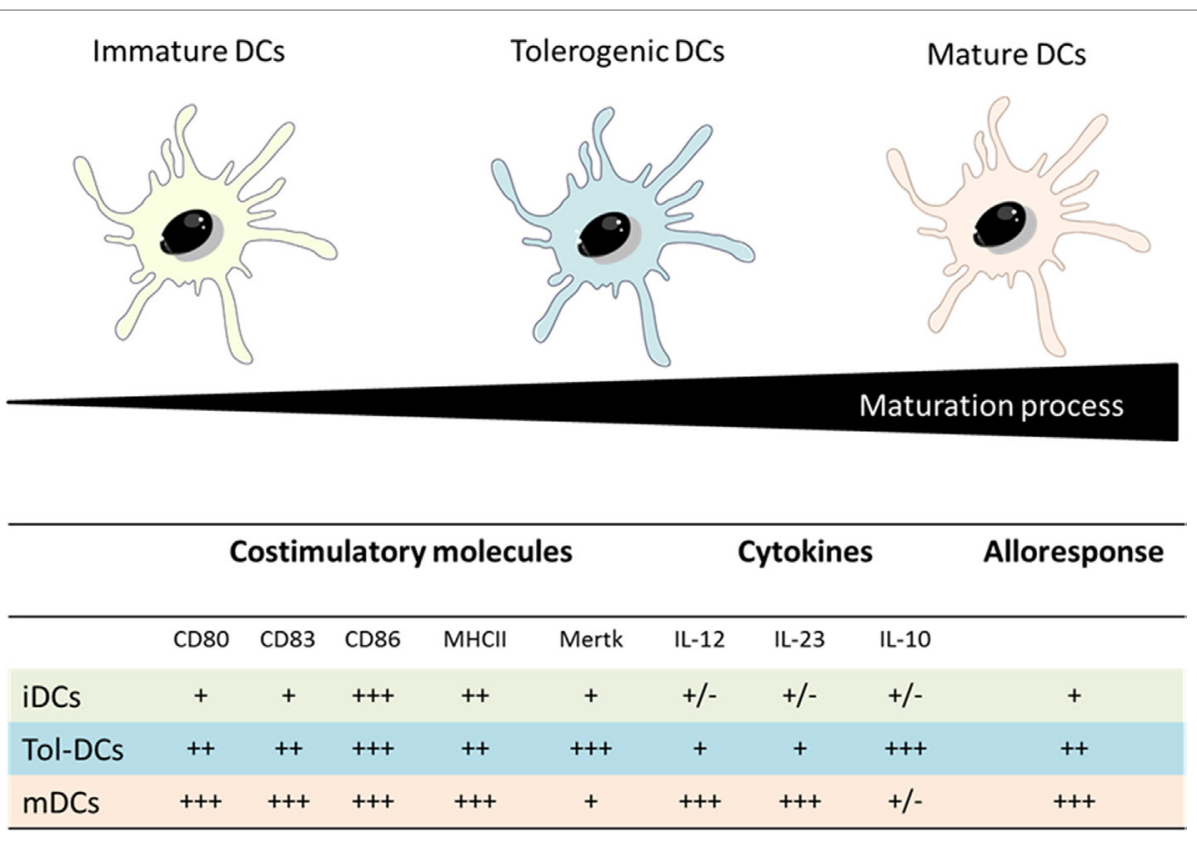

FIGURE 5 | Comparison between iDCs, tolerogenic DCs and mature DCs properties. Adapted from: Hubo et al. (59).

In summary, Tol-DCs generated ex vivo using immunosuppressive agents, induced $\mathrm{T}$ regulatory cells through different mechanism such as lower expression of co stimulatory molecules, expression of inhibitory receptors and IL-10 production.

\section{Tol-DC Therapy in the Animal Model of MS}

Animal models are the first step in the development of new therapies, and antigen-specific therapies are not an exception to this rule. Over the past several decades animal models have been used to understand different aspects of human MS. There are three different animal models of MS that are the most commonly used: (1) the experimental autoimmune encephalomyelitis (EAE), (2) viral induced models, and (3) toxin-induced models of demyelination (6).

In addition to the in vitro demonstration of the capacity of Tol-DC to induce immune tolerance, the role of Tol-DCs has been evaluated in the EAE model. The critical role of mDCs and pDCs in the chronic pathogenesis of EAE in Lewis rats described by Miller and colleagues makes this model extremely relevant to study positive and negative regulatory pathways involved in MS and other chronic autoimmune diseases (64). Wang et al. demonstrated the involvement of $\mathrm{CD}_{11 \mathrm{~b}^{+}}$and CD11 $\mathrm{c}^{+} \mathrm{DCs}$ in the generation of both T-regs and Tr1 cells, by depleting DCs they observed that tolerance effect disappeared (65). In consequence, the induction of DCs with a regulatory profile is a key mechanism underlying auto antigen-induced tolerance (64). It is interesting to highlight that studies performed in EAE induced in Lewis rats demonstrated that the maturation state as well as the route of administration influence on the induction of tolerance by these DCs which is in concordance with the in vitro performed studies $(65,66)$. Moreover, different authors have described that the administration of Tol-DCs generated with different immunosuppressive agents such as vitD3 or estriol induced a decrease of the incidence of the disease as well as they promoted the induction of regulatory T-cells though higher levels of IL-10 production $(63,67)$.

In addition, other authors have performed comparisons regarding the use of immunosuppressive oral drugs such as vitD3 and (for 20 days after EAE induction) or pretreating DCs before EAE induction. The results obtained were similar in both cases: significant improvement of clinical severity and an increase of regulatory $\mathrm{CD} 4^{+} \mathrm{Foxp}^{+}$cells and increased IL- 10 levels in lymph nodes from treated animals suggesting that DCs are the main target of tolerogenic effect of vitamin D. Some studies pointed out that in the absence of DCs during the priming process of autoreactive T-cells leads to a unidirectional deficiency of cell generation which results in a fulminant attack against CNS $(65,66,68)$. Different studies using DCs to induce tolerance have been performed in EAE animal models of mice and rats and they are summarized in Table 3.

In addition, Tol-DCs have also been generated for another disease models such as type I diabetes T1D by using a combination of both dexamethasone and vitD3. This generated Tol-DCs presented a stable phenotype and a high capacity to induce T-reg cells (73). Moreover, other protocols, such as DC treatment with CD40, CD80, and CD86 antisense oligo nucleotides or even low doses of GM-CSF has also been reported although in some cases partial loss of tolerance have been reported.

The critical part is that after being culture, all generated Tol-DCs have to present different characteristics: (a) low levels of co stimulatory molecules, (b) stability when challenges with maturation stimuli and also produce IL-10, (c) lower activation of T-cells (73).

Overall, different protocols for Tol-DCs in preclinical studies has been shown to be beneficious to treat different autoimmune 
TABLE 3 | Summary of tolerogenic DCs therapy in animal models $(65,67,69,70)$.

\begin{tabular}{|c|c|c|c|}
\hline Animal model & Dendritic cells injected & Route of administration & Reference \\
\hline EAE in C57BL/6 mice & $1 \times 10^{6}$ & Intravenous & Leng et al. (67) \\
\hline EAE in C57BL/6J mice & $1 \times 10^{6}$ & Intravenous & Mansilla et al. (63) \\
\hline EAE in C57BL/6 mice & $1-2 \times 10^{6}$ to $8-10 \times 10^{6}$ & Intravenous & Papenfuss et al. (67) \\
\hline EAE in C57BL/6 mice & $5 \times 10^{5}$ & Subcutaneous or intraperitoneally & Aghdami et al. (71) \\
\hline EAE in Lewis rats & $2 \times 10^{6}$ & Subcutaneous or intravenous & Zhang et al. (72) \\
\hline EAE in Lewis rats & $1 \times 10^{6}$ & Subcutaneous & Xiao et al. (66) \\
\hline
\end{tabular}

EAE, experimental autoimmune encephalomyelitis.

diseases, in particular for EAE induction the use of vitD3 or corticosteroids is the most extended.

\section{Therapeutic Application of Tol-DCs in Type I Diabetes, RA and Crohn's Disease}

Following the encouraging results obtained from different in vitro and preclinical studies in animal models, Tol-DCs are revealed as a promising therapy for autoimmune diseases and transplantation (32). Consequently, in 2011, the first phase I clinical trial with Tol-DCs was conducted at the University of Pittsburgh. The trial enrolled 10 insulin-dependent diabetic patients, and administrated control DCs to three patients and immunosuppressive DC (iRNA for CD40, CD80, and for CD86) to seven patients. The treatment was safe and well tolerated. There were no changes in insulin requirements, hematology assessments or blood immune cell population levels in both groups, showing a slight increase of $\mathrm{CD}^{+} \mathrm{CD} 25^{+++}$FoxP $^{+} \mathrm{T}$ cells in immunosuppressive DC group. All treated patients had normal immune responses to vaccination and alloantigen stimulation in vitro (74). Thus, a double-blinded, placebo-controlled cross-over phase II trial is planned to start in Diabetes mellitus type 1 in 24 patients with a recent onset of the disease, inducing tolerability of DC with antisense DNA targeting CD40, CD80, and CD86 (NCT02354911).

Among autoimmune arthritis, two trials have been published recently. In the first one, a unique intradermal administration of "Rheumavax" (autologous DCs modified with a nuclear factor $\kappa \mathrm{b}$ inhibitor exposed to 4-citrullinated peptide antigens), was studied in a phase I clinical trial of RA patients. They observed a significant increased ratio of regulatory to effector $\mathrm{T}$ cells and a reduction of IL-15, which is a relevant pro-inflammatory cytokine. Moreover, in a more clinical level they found a decrease of DAS28 which is a clinical scale for RA severity together with no disease flares (75). Furthermore, in 2017, results from AUTODECRA trial (NCT01352858) came out resulting a safe and well tolerated therapy with no target knee flares, but with no significant clinical and immunomodulatory changes in serum (76).

Importantly, other clinical trials have been recently reported in other autoimmune diseases such as Crohn's disease. In Crohn's disease, our institution conducted a phase I clinical trial to demonstrate the safety of intraperitoneal administration of autologous Tol-DCs in refractory patients. The immune monitoring studies showed an increase of circulating T-regs and a decrease of IFN- $\gamma$ production after T-cell activation (31). Regarding organ transplantation, two trials are ongoing. A phase I clinical trial, open-label and non-controlled, in liver transplantation is aimed to assess the safety of Tol-DCs therapy in this type of patients
(NCT03164265). The ONEatDC study, aims to assess if TolDC administration before renal transplantation is beneficial to reduce immunosuppression needs (NCT02252055).

Overall, the encouraging results obtained in above mentioned clinical trials, of an increase immunosuppressive activity, drawn Tol-DCs as a potential tool to modulate autoinflammatory diseases in the coming years.

\section{ANTIGEN-SPECIFIC THERAPIES IN MS AND NMO}

In the recent years, several strategies to modulate antigenspecific T-cells have been evaluated in therapeutic clinical trials for patients with MS and NMO. Among the advantages to use antigen-specific therapies, they lack of general immunosuppression and its side effects as infections and cancer, as well as the lack of metabolic activity that activates self-reactive $\mathrm{T}$ cells, the induction of tolerance to a specific antigen without changing the general immunity (77). The use of DC to induce immune tolerance is also pursuit in patients with MS and NMO. In this sense, a phase I trial to assess the safety of Tol-DC in MS and NMO patients in an ascending dose of intravenous administration of the DCs (NCT02283671) has been performed at our institution and the results are under evaluation. In addition, two more clinical trials are ongoing (NCT02618902) and (NCT02903537), which will provide precious information about safety, modulation of immune response and clinical efficacy.

Several approaches to induce antigen-specific tolerization have been evaluated as DNA vaccination of myelin protein, peptides inoculation, altered peptide ligand (APL) administration to modify TCR recognition, autologous myelin-reactive $\mathrm{T}$ cells administration, HLA/MOG recombinant construct administration and autologous PBMCs coupled with myelin-peptides administration, Tol-DCs with myelin-peptides administration (78). Specifically, myelin-peptides approaches are based in a myelin relevant immunodominant peptide administration, like administration of the synthetic peptide itself like MBP, MOG, or PLP, administration of APL or the administration of a region of TCR-peptide complex.

Antigen-specific therapeutic approaches have been demonstrated in the majority of the phase I clinical trials to be safe and well tolerated. However, a trial conducted at NIH with APL induced disease exacerbation and the trial was stopped due to safety issues (79). The concept of APL is based in the administration of modified peptides by introducing some amino acids in substitution in specific positions relevant to link with the TCR, but 
without changing the MHC binding part. This strategy is aimed to inhibit the inflammatory $\mathrm{T}$ cell response, as acts as partial agonist or as antagonist. A phase II trial using $\mathrm{MBP}_{83-99}$ was interrupted as three out of eight participants presented relapses during the clinical trial, that were considered as inflammatory activation as MRI controls showed disease worsening, and this was correlated with MBP specific T cell expansion in blood and CSF samples (80). Two more trials with APLs were done afterward, without objectifying exacerbations of the disease activity (81).

DNA vaccination aims to induce tolerance using heterotopic expression of some antigens, for example using whole human MBP protein. The BHT-3009 molecule is a union of the whole MBP molecule, a human cytomegalovirus promoter and an altered plasmid. In two clinical trials it was demonstrated safe and gadolinium-enhancing lesions were fewer in the treated groups comparing with placebo groups; although, there were significant improvement in clinical outcomes. Immunologically, a decrease in IFN- $\gamma$ production and T cell proliferation by MBP, PLP, and MOG specific T-cells was observed (82). In another trial, reduction of autoreactive $\mathrm{T}$ cells was demonstrated with this approach, creating a proof of concept of the possible efficacy of DNA vaccination (80).

The vaccination with $\mathrm{T}$-cell consists in the administration of activated and irradiated MPB-specific T-cell lines and clones (attenuated autologous T-cells). Phase I and phase II clinical trials have been done, with no relevant side effects, but without significant clinical improvement in treated group comparing with placebo group (83).

Other antigen-specific tolerization approach studied in MS was the antigen-coupled cell tolerance, based on inactivated autologous PBMCs chemically linked with myelin relevant peptides. After proving reduction of onset and severity as well as preventing epitope spreading in EAE, this approach was evaluated in humans. In 2013, a phase I clinical trial (ETIMS trial) was published where antigen-specific tolerance induced with inactivated PBMCs coupled with six immunodominant myelin-peptides was safe, with some immunological promising results to objectify clinical significance (78). Significant advantage of this approach is that the tolerization to several myelin relevant peptides derived from three different antigens (MBP, MOG, and PLP) simultaneously is aimed to prevent the epitope spreading situation.

\section{REFERENCES}

1. Jacques FH. Defining the clinical course of multiple sclerosis: the 2013 revisions. Neurology (2015) 84:963-963. doi:10.1212/01.wnl.0000462309.76486.c5

2. Pugliatti M, Rosati G, Carton H, Riise T, Drulovic J, Vecsei L, et al. The epidemiology of multiple sclerosis in Europe. Eur J Neurol (2006) 13:700-22. doi:10.1111/j.1468-1331.2006.01342.x

3. Kotelnikova E, Kiani NA, Abad E, Martinez-Lapiscina EH, Andorra M, Zubizarreta I, et al. Dynamics and heterogeneity of brain damage in multiple sclerosis. PLoS Comput Biol (2017) 13:e1005757. doi:10.1371/journal. pcbi. 1005757

4. Polman CH, Reingold SC, Banwell B, Clanet M, Cohen JA, Filippi M, et al. Diagnostic criteria for multiple sclerosis: 2010 revisions to the McDonald criteria. Ann Neurol (2011) 69(2):292-302. doi:10.1002/ana.22366

5. Ransohoff RM, Hafler DA, Lucchinetti CF. Multiple sclerosis - a quiet revolution. Nat Rev Neurol (2015) 11:134-42. doi:10.1038/nrneurol.2015.14
To synthetize, there are different antigen-specific therapies that have been asses in MS patients. The majority has been presented as safe and well tolerated with encouraging data regarding the clinical benefits.

\section{CONCLUSION AND FUTURE PERSPECTIVES}

Antigen-specific tolerance in autoimmune diseases is a therapeutic approach that is currently been evaluated in MS and NMO as well as in other autoimmune diseases. Different reports have demonstrated that DCs are powerful therapeutic tools to modify the immune response and restore the immune tolerance in animal models and in preclinical data. Most importantly, the use of Tol-DCs in clinical trials is being safe in several phase I clinical trials (type I Diabetes, RA and Crohn's disease) showing in some of the studies promising clinical and immunomodulatory results.

In MS several reports have revealed the therapeutic effect of Tol-DCs in ameliorating EAE in animal model. These results highlight the importance of DCs in the homeostasis control and open new avenues for an innovative therapeutic indication for human patients. A major challenge is to translate all these results obtained in animal models to humans. For that reason, it will be crucial to correlate clinical efficacy with modulation of immunological parameters and also to define the optimal administration route, dose of cells, tolerogenic treatments and the potential tolerogenic effect of circulating DCs.

From the studies conducted so far, several important considerations have been raised, application of Tol-DCs in humans is safe and well tolerated without remarkable side effects and showing promising immunological and clinical results. However, phase II and/or III clinical trials including control (placebo) group will bring some light about the clinical efficacy of this therapy in MS/ NMO patients. In addition, more studies are needed to evaluate the real effectiveness and the possibility to use Tol-DC as a real treatment for autoimmune diseases.

\section{AUTHOR CONTRIBUTIONS}

GF-G, IZ, and RC wrote the manuscript and designed figures. PV and $\mathrm{DB}-\mathrm{R}$ revised the manuscript.

6. Denic A, Johnson AJ, Bieber AJ, Warrington AE, Rodriguez M, Pirko I. The relevance of animal models in multiple sclerosis research. Pathophysiolgy (2011) 18:21-9. doi:10.1016/j.pathophys.2010.04.004

7. Dos Passos GR, Sato DK, Becker J, Fujihara K. Th17 cells pathways in multiple sclerosis and neuromyelitis optica spectrum disorders: pathophysiological and therapeutic implications. Mediators Inflamm (2016) 2016:5314541. doi:10.1155/2016/5314541

8. Rostami A, Ciric B. Role of Th17 cells in the pathogenesis of CNS inflammatory demyelination. J Neurol Sci (2013) 333:76-87. doi:10.1016/j.jns.2013.03.002

9. Fletcher JM, Lalor SJ, Sweeney CM, Tubridy N, Mills KHG. T cells in multiple sclerosis and experimental autoimmune encephalomyelitis. Clin Exp Immunol (2010) 162:1-11. doi:10.1111/j.1365-2249.2010.04143.x

10. Murphy ÁC, Lalor SJ, Lynch MA, Mills KHG. Infiltration of Th1 and Th17 cells and activation of microglia in the CNS during the course of experimental autoimmune encephalomyelitis. Brain Behav Immun (2010) 24:641-51. doi:10.1016/j.bbi.2010.01.014 
11. Mondal S, Roy A, Pahan K. Functional blocking monoclonal antibodies against IL-12p40 homodimer inhibit adoptive transfer of experimental allergic encephalomyelitis. J Immunol (2009) 182:5013-23. doi:10.4049/ jimmunol.0801734

12. Constantinescu CS, Farooqi N, O'Brien K, Gran B. Experimental autoimmune encephalomyelitis (EAE) as a model for multiple sclerosis (MS). BrJ Pharmacol (2011) 164:1079-106. doi:10.1111/j.1476-5381.2011.01302.x

13. Goverman J. Autoimmune $\mathrm{T}$ cell responses in the central nervous system. Nat Rev Immunol (2009) 9:393-407. doi:10.1038/nri2550

14. Sato DK, Callegaro D, Lana-Peixoto MA, Waters PJ, de Haidar Jorge FM, Takahashi T, et al. Distinction between MOG antibody-positive and AQP4 antibody-positive NMO spectrum disorders. Neurology (2014) 82:474-81. doi:10.1212/WNL.0000000000000101

15. Marrie RA, Gryba C. The incidence and prevalence of neuromyelitis optica. Int J MS Care (2013) 15(3):113-8. doi:10.7224/1537-2073.2012-048

16. Sepulveda M, Aldea M, Escudero D, Llufriu S, Arrambide G, Otero-Romero S, et al. Epidemiology of NMOSD in Catalonia: influence of the new 2015 criteria in incidence and prevalence estimates. Mult Scler (2017) 1:1352458517735191. doi:10.1177/1352458517735191

17. Papadopoulos MC, Verkman AS. Aquaporin 4 and neuromyelitis optica. Lancet Neurol (2012) 11(6):535-44. doi:10.1016/S1474-4422(12)70133-3

18. Mariotto S, Ferrari S, Monaco S, Benedetti MD, Schanda K, Alberti D, et al. Clinical spectrum and IgG subclass analysis of anti-myelin oligodendrocyte glycoprotein antibody-associated syndromes: a multicenter study. J Neurol (2017) 264:2420-30. doi:10.1007/s00415-017-8635-4

19. Scolding N, Barnes D, Cader S, Chataway J, Chaudhuri A, Coles A, et al. Association of British Neurologists: revised (2015) guidelines for prescribing disease-modifying treatments in multiple sclerosis. Pract Neurol (2015) 15(4):273-9. doi:10.1136/practneurol-2015-001139

20. Papadopoulos MC, Bennett JL, Verkman AS. Treatment of neuromyelitis optica: state-of-the-art and emerging therapies. Nat Rev Neurol (2014) 10(9):493-506. doi:10.1038/nrneurol.2014.141

21. Kleiter I, Gold R. Present and future therapies in neuromyelitis optica spectrum disorders. Neurotherapeutics (2016) 13:70-83. doi:10.1007/s13311015-0400-8

22. Saadoun S, Papadopoulos M, Davies D, Bell B, Krishna S. Increased aquaporin 1 water channel expression in human brain tumours. Br J Cancer (2002) 87: 621-3. doi:10.1038/sj.bjc.6600512

23. Kohm AP, Turley DM, Miller SD. Targeting the TCR: T-cell receptor and peptide-specific tolerance-based strategies for restoring self-tolerance in CNS autoimmune disease. Int Rev Immunol (2005) 24:361-92. doi:10.1080/ 08830180500371207

24. Boltjes A, van Wijk F. Human dendritic cell functional specialization in steady-state and inflammation. Front Immunol (2014) 5:131. doi:10.3389/ fimmu.2014.00131

25. Moser M. Dendritic cells in immunity and tolerance-do they display opposite functions? Immunity (2003) 19:5-8. doi:10.1016/S1074-7613(03)00182-1

26. O'Neill DW. Manipulating dendritic cell biology for the active immunotherapy of cancer. Blood (2004) 104(8):2235-46. doi:10.1182/blood-2003-12-4392

27. Kaka AS, Foster AE, Weiss HL, Rooney CM, Leen AM. Using dendritic cell maturation and IL-12 producing capacity as markers of function: a cautionary tale. J Immunother (2008) 31:359-69. doi:10.1097/CJI.0b013e318165f5d2

28. Kapsenberg ML. Dendritic-cell control of pathogen-driven T-cell polarization. Nat Rev Immunol (2003) 3:984-93. doi:10.1038/nri1246

29. Steinman RM, Nussenzweig MC. Avoiding horror autotoxicus: The importance of dendritic cells in peripheral T cell tolerance. Proc Natl Acad Sci U S A (2002) 99(1):351-8. doi:10.1073/pnas.231606698

30. Cabezón R, Benítez-Ribas D. Therapeutic potential of tolerogenic dendritic cells in IBD: from animal models to clinical application. Clin Dev Immunol (2013) 2013:789814. doi:10.1155/2013/789814

31. Jauregui-Amezaga A, Cabezón R, Ramírez-Morros A, España C, Rimola J, Bru C, et al. Intraperitoneal administration of autologous tolerogenic dendritic cells for refractory Crohn's disease: a phase I study. J Crohn's Colitis (2015) 9: 1071-8. doi:10.1093/ecco-jcc/jjv144

32. Suwandi JS, Toes REM, Nikolic T, Roep BO. Inducing tissue specific tolerance in autoimmune disease with tolerogenic dendritic cells. Clin Exp Rheumatol (2015) 33:S97-103.

33. Mildner A, Jung S. Development and function of dendritic cell subsets. Immunity (2014) 40:642-56. doi:10.1016/j.immuni.2014.04.016
34. Collin M, McGovern N, Haniffa M. Human dendritic cell subsets. Immunology (2013) 140:22-30. doi:10.1111/imm.12117

35. Nirschl CJ, Anandasabapathy N. Duality at the gate: skin dendritic cells as mediators of vaccine immunity and tolerance. Hum Vaccin Immunother (2016) 12:104-16. doi:10.1080/21645515.2015.1066050

36. Haniffa M, Gunawan M, Jardine L. Human skin dendritic cells in health and disease. J Dermatol Sci (2015) 77:85-92. doi:10.1016/j.jdermsci.2014.08.012

37. Cohn L, Delamarre L. Dendritic cell-targeted vaccines. Front Immunol (2014) 5:255. doi:10.3389/fimmu.2014.00255

38. Bakdash G, Buschow SI, Gorris MAJ, Halilovic A, Hato SV, Sköld AE, et al. Expansion of a BDCA1+CD14+ myeloid cell population in melanoma patients may attenuate the efficacy of dendritic cell vaccines. Cancer Res (2016) 76:4332-46. doi:10.1158/0008-5472.CAN-15-1695

39. Swiecki M, Colonna M. The multifaceted biology of plasmacytoid dendritic cells. Nat Rev Immunol (2015) 15:471-85. doi:10.1038/nri3865

40. Jin J-O, Zhang W, Du JY, Yu Q. BDCA1-positive dendritic cells (DCs) represent a unique human myeloid DC subset that induces innate and adaptive immune responses to Staphylococcus aureus infection. Infect Immun (2014) 82:4466-76. doi:10.1128/IAI.01851-14

41. Steinman RM, Pope M. Exploiting dendritic cells to improve vaccine efficacy. J Clin Invest (2002) 109:1519-26. doi:10.1172/JCI0215962

42. Romani N, Clausen BE, Stoitzner P. Langerhans cells and more: langerinexpressing dendritic cell subsets in the skin. Immunol Rev (2010) 234:120-41. doi:10.1111/j.0105-2896.2009.00886.x

43. MacDonald KPA, Munster DJ, Clark GJ, Dzionek A, Schmitz J, Hart DNJ. Characterization of human blood dendritic cell subsets. Blood (2002) 100: 4512-20. doi:10.1182/blood-2001-11-0097

44. Ohradanova-Repic A, Machacek C, Fischer MB, Stockinger H. Differentiation of human monocytes and derived subsets of macrophages and dendritic cells by the HLDA10 monoclonal antibody panel. Clin Transl Immunology (2016) 5:1-9. doi:10.1038/cti.2015.39

45. Li H, Shi B. Tolerogenic dendritic cells and their applications in transplantation. Cell Mol Immunol (2015) 12:24-30. doi:10.1038/cmi.2014.52

46. Macri C, Dumont C, Johnston APR, Mintern JD. Targeting dendritic cells: a promising strategy to improve vaccine effectiveness. Clin Transl Immunology (2016) 5:e66. doi:10.1038/cti.2016.6

47. Chung CYJ, Ysebaert D, Berneman ZN, Cools N. Dendritic cells: cellular mediators for immunological tolerance. Clin Dev Immunol (2013) 2013 972865. doi:10.1155/2013/972865

48. Ahmed MS, Bae Y-S. Dendritic cell-based immunotherapy for rheumatoid arthritis: from bench to bedside. Immune Netw (2016) 16:44-51. doi:10.4110/ in.2016.16.1.44

49. Kirkwood JM, Butterfield LH, Tarhini AA, Zarour H, Kalinski P, Ferrone S. Immunotherapy of cancer in 2012. CA Cancer J Clin (2012) 62:309-35. doi:10.3322/caac. 20132

50. Xue Z, Zhang X, Chen M, Lu X, Deng R, Ma Y. Dendritic cells transduced with single immunoglobulin IL-1-related receptor exhibit immature properties and prolong islet allograft survival. Front Immunol (2017) 8:1671. doi:10.3389/ fimmu.2017.01671

51. Sharpe AH, Freeman GJ. The B7-CD28 superfamily. Nat Rev Immunol (2002) 2:116-26. doi:10.1038/nri727

52. Ganguly D, Haak S, Sisirak V, Reizis B. The role of dendritic cells in autoimmunity. Nat Rev Immunol (2013) 13:566-77. doi:10.1038/nri3477

53. Agrawal S, Ganguly S, Hajian P, Cao J-N, Agrawal A. PDGF upregulates CLEC-2 to induce T regulatory cells. Oncotarget (2015) 6:28621-32. doi:10.18632/ oncotarget. 5765

54. Hackstein H, Thomson AW. Dendritic cells: emerging pharmacological targets of immunosuppressive drugs. Nat Rev Immunol (2004) 4:24-35. doi:10.1038/ nri1256

55. van Kooten C, Stax AS, Woltman AM, Gelderman KA. Handbook of experimental pharmacology "dendritic cells," the use of dexamethasone in the induction of tolerogenic DCs. Handb Exp Pharmacol (2009):233-49.

56. Kalantari T, Kamali-Sarvestani E, Ciric B, Karimi MH, Kalantari M, Faridar A, et al. Generation of immunogenic and tolerogenic clinical-grade dendritic cells.Immunol Res (2011) 51:153-60. doi:10.1007/s12026-011-8255-5

57. García-González P, Morales R, Hoyos L, Maggi J, Campos J, Pesce B, et al. A short protocol using dexamethasone and monophosphoryl lipid A generates tolerogenic dendritic cells that display a potent migratory capacity to lymphoid chemokines. J Transl Med (2013) 11:1-15. doi:10.1186/1479-5876-11-128 
58. Cabezón R, Ricart E, España C, Panés J, Benitez-Ribas D. Gram-negative enterobacteria induce tolerogenic maturation in dexamethasone conditioned dendritic cells. PLoS One (2012) 7(1-13):e52456. doi:10.1371/journal.pone. 0052456

59. Hubo M, Trinschek B, Kryczanowsky F, Tuettenberg A, Steinbrink K, Jonuleit $\mathrm{H}$. Costimulatory molecules on immunogenic versus tolerogenic human dendritic cells. Front Immunol (2013) 4:82. doi:10.3389/fimmu.2013. 00082

60. Sun G, Shan J, Li Y, Feng L, Zhou Y, Guo Y, et al. Adoptive infusion of tolerance dendritic cells prolongs survival of small intestine allografts in rats: systematic review and meta-analysis. J Evid Based Med (2013) 6(3):185-96. doi:10.1111/jebm.12050

61. Hu J, Wan Y. Tolerogenic dendritic cells and their potential applications. Immunology (2011) 132:307-14. doi:10.1111/j.1365-2567.2010.03396.x

62. Raïch-Regué D, Grau-López L, Naranjo-Gómez M, Ramo-Tello C, PujolBorrell R, Martínez-Cáceres E, et al. Stable antigen-specific T-cell hyporesponsiveness induced by tolerogenic dendritic cells from multiple sclerosis patients. Eur J Immunol (2012) 6(3):185-96. doi:10.1002/eji.201141835

63. Mansilla MJ, Sellès-Moreno C, Fàbregas-Puig S, Amoedo J, Navarro-Barriuso J, Teniente-Serra A, et al. Beneficial effect of tolerogenic dendritic cells pulsed with MOG autoantigen in experimental autoimmune encephalomyelitis. CNS Neurosci Ther (2015) 21:222-30. doi:10.1111/cns.12342

64. Li H, Zhang G-X, Chen Y, Xu H, Fitzgerald DC, Zhao Z, et al. CD11c+CD11b+ dendritic cells play an important role in intravenous tolerance and the suppression of experimental autoimmune encephalomyelitis. J Immunol (2008) 181:2483-93. doi:10.4049/jimmunol.181.4.2483

65. Wang L, Li Z, Ciric B, Safavi F, Zhang G-X, Rostami A. Selective depletion of $\mathrm{CD} 11 \mathrm{c}(+) \mathrm{CD} 11 \mathrm{~b}(+)$ dendritic cells partially abrogates tolerogenic effects of intravenous MOG in murine EAE. Eur J Immunol (2016) 46:2454-66. doi:10.1002/eji.201546274

66. Xiao BG, Huang YM, Yang JS, Xu LY, Link H. Bone marrow-derived dendritic cells from experimental allergic encephalomyelitis induce immune tolerance to EAE in Lewis rats. Clin Exp Immunol (2001) 125:300-9. doi:10.1046/j.1365-2249.2001.01573.x

67. Papenfuss TL, Powell ND, McClain MA, Bedarf A, Singh A, Gienapp IE, et al. Estriol generates tolerogenic dendritic cells in vivo that protect against autoimmunity. J Immunol (2011) 186:3346-55. doi:10.4049/jimmunol. 1001322

68. Xie Z-X, Zhang H-L, Wu X-J, Zhu J, Ma D-H, Jin T. Role of the immunogenic and tolerogenic subsets of dendritic cells in multiple sclerosis. Mediators Inflamm (2015) 2015:1-20. doi:10.1155/2015/513295

69. Naranjo-Gómez M, Raïch-Regué D, Oñate C, Grau-López L, Ramo-Tello C, Pujol-Borrell R, et al. Comparative study of clinical grade human tolerogenic dendritic cells. J Transl Med (2011) 9:1-14. doi:10.1186/1479-5876-9-89

70. Turley DM, Miller SD. Prospects for antigen-specific tolerance based therapies for the treatment of multiple sclerosis. Results Probl Cell Differ (2010) 51:217-35. doi:10.1007/400_2008_13

71. Aghdami N, Gharibdoost F, Moazzeni SM. Experimental autoimmune encephalomyelitis (EAE) induced by antigen pulsed dendritic cells in the C57BL/6 mouse: influence of injection route. Exp Anim (2008) 57(1):45-55.

72. Zhang X, Koldzic DN, Izikson L, Reddy J, Nazareno RF, Sakaguchi S, et al. IL-10 is involved in the suppression of experimental autoimmune encephalomyelitis by $\mathrm{CD} 25^{+} \mathrm{CD} 4^{+}$regulatory T cells. Int Immunol (2004) 16(2):249-56. doi:10.1093/intimm/dxh029
73. Spísek R, Palová-Jelínková L, Petruzelková L, Sedivá A, Fundová P, Pruhová K et al. Tolerogenic dendritic cells from poorly compensated type 1 diabetes patients have decreased ability to induce stable antigen-specific T cell hyporesponsiveness and generation of suppressive regulatory T cells. J Immunol (2016). Available from: http://www.jimmunol.org/content/early/2016/12/07/ jimmun (Accessed: April 24, 2018).

74. Giannoukakis N, Phillips B, Finegold D, Harnaha J, Trucco M. Phase I (safety) study of autologous tolerogenic dendritic cells in type 1 diabetic patients. Diabetes Care (2011) 34:2026-32. doi:10.2337/dc11-0472

75. Benham H, Nel HJ, Law SC, Mehdi AM, Street S, Ramnoruth N, et al. Citrullinated peptide dendritic cell immunotherapy in HLA risk genotypepositive rheumatoid arthritis patients. Sci Transl Med (2015) 7:ra87-290. doi:10.1126/scitranslmed.aaa9301

76. Bell GM, Anderson AE, Diboll J, Reece R, Eltherington O, Harry RA, et al. Autologous tolerogenic dendritic cells for rheumatoid and inflammatory arthritis. Ann Rheum Dis (2017) 76:227-34. doi:10.1136/annrheumdis-2015-208456

77. Kim T-G, Kim DS, Kim H-P, Lee M-G. The pathophysiological role of dendritic cell subsets in psoriasis. BMB Rep (2014) 47:60-8. doi:10.5483/ BMBRep.2014.47.2.014

78. Lutterotti A, Yousef S, Sputtek A, Sturner KH, Stellmann J-P, Breiden P, et al. Antigen-specific tolerance by autologous myelin peptide-coupled cells: a phase 1 trial in multiple sclerosis. Sci Transl Med (2013) 5:1-11. doi:10.1126/ scitranslmed.3006168

79. Sospedra M, Martin R. Immunology of multiple sclerosis. Semin Neurol (2016) 36:115-27. doi:10.1055/s-0036-1579739

80. Medaer R, Stinissen P, Truyen L, Raus J, Zhang J. Depletion of myelinbasic-protein autoreactive $\mathrm{T}$ cells by T-cell vaccination: pilot trial in multiple sclerosis. Lancet (London, England). (1995) 346:807-8. doi:10.1016/S01406736(95)91622-9

81. Edwards LJ, Evavold BD. T cell recognition of weak ligands: roles of signaling, receptor number, and affinity. Immunol Res (2011) 50(1):39-48. doi:10.1007/ s12026-011-8204-3

82. Ifergan I, Wosik K, Cayrol R, Kébir H, Auger C, Bernard M, et al. Statins reduce human blood-brain barrier permeability and restrict leukocyte migration: relevance to multiple sclerosis. Ann Neurol (2006) 60:45-55. doi:10.1002/ ana. 20875

83. Karussis D, Shor H, Yachnin J, Lanxner N, Amiel M, Baruch K, et al. T cell vaccination benefits relapsing progressive multiple sclerosis patients: a randomized, double-blind clinical trial. PLoS One (2012) 7:e50478. doi:10.1371/ journal.pone.0050478

Conflict of Interest Statement: PV is an employee of Genentech. The remaining authors declare that the research was conducted in the absence of any commercial or financial relationships that could be construed as a potential conflict of interest.

The reviewer IL-P and handling Editor declared their shared affiliation.

Copyright (C) 2018 Flórez-Grau, Zubizarreta, Cabezón, Villoslada and Benitez-Ribas. This is an open-access article distributed under the terms of the Creative Commons Attribution License (CC BY). The use, distribution or reproduction in other forums is permitted, provided the original author(s) and the copyright owner are credited and that the original publication in this journal is cited, in accordance with accepted academic practice. No use, distribution or reproduction is permitted which does not comply with these terms. 